\title{
Globe
}

Revue internationale d'études québécoises

\section{Janet M. Paterson : Figures de l'Autre dans le roman québécois. Québec, Nota bene, 2004}

\section{Anthony Purdy}

Volume 9, numéro 1, 2006

URI : https://id.erudit.org/iderudit/1000806ar

DOI : https://doi.org/10.7202/1000806ar

Aller au sommaire du numéro

\section{Éditeur(s)}

Globe, Revue internationale d'études québécoises

ISSN

1481-5869 (imprimé)

1923-8231 (numérique)

Découvrir la revue

Citer ce compte rendu

Purdy, A. (2006). Compte rendu de [Janet M. Paterson : Figures de l'Autre dans le roman québécois. Québec, Nota bene, 2004]. Globe, 9(1), 275-280.

https://doi.org/10.7202/1000806ar d'utilisation que vous pouvez consulter en ligne.

https://apropos.erudit.org/fr/usagers/politique-dutilisation/ 


\section{Recensions}

\section{Janet M. Paterson}

Figures de l'Autre dans le roman québécois.

Québec, Nota bene, 2004.

Cette belle étude a pour objet la représentation du personnage Autre $^{1}$ dans le roman québécois. S'échelonnant sur une période de 135 ans - des Anciens Canadiens de Philippe Aubert de Gaspé (1863) à La petite fille qui aimait trop les allumettes de Gaétan Soucy (1998) en passant par des textes aussi variés que Le survenant de Germaine Guèvremont, Kamouraska d'Anne Hébert, Volkswagen blues de Jacques Poulin, La Québécoite de Régine Robin et Le pavillon des miroirs de Sergio Kokis -, les romans analysés par Janet Paterson avec sa clarté habituelle balisent un parcours historique non seulement de la figure de l'Autre, mais aussi, en filigrane et par le biais de l'altérité, de toute une société. Les analyses sont d'ailleurs sobres et mesurées, introduisant un ton calme et raisonnable dans un domaine, celui de l'identitaire, où le polémique et le passionnel règnent souvent.

Un premier chapitre théorique commence par poser l'altérité comme concept rigoureusement relationnel, et l'Autre comme formation discursive et culturelle qu'il faut éviter d'hypostasier. Empruntant à Éric Landowski la notion de groupe de référence, Paterson définit l'Autre comme un écart par rapport à une unité plus grande, elle-même souvent diffuse et mouvante. D'où la nécessité de distinguer les notions d'altérité et de différence ; pour passer de celle-ci à celle-là, il faut qu'un * groupe de référence, habituellement le groupe dominant, fixe l'inventaire des traits différentiels qui serviront à construire les "figures de l'Autre"; construction qui produit souvent des systèmes de ségrégation " (p. 25).

1. Paterson se sert indifféremment de deux désignations : "le personnage Autre ", - le personnage de l'Autre *.

Karim Larose [éd.], • Recensions =, Globe. Revue internationale d'études québécoises, vol. 9, $\mathrm{n}^{\circ} 1,2006$. 


\section{REVUE INTERNATIONALE D'ÉTUDES QUÉBÉCOISES}

En tant que formation discursive (et idéologique), la figure de l'Autre est " variable, mouvante et susceptible de renversements. Elle n'est marquée d'aucune immanence et peut être dotée de traits positifs ou négatifs " (p. 27).

Quels sont donc les facteurs discursifs qui participent à une telle construction de l'altérité ? Paterson en énumère plusieurs : l'énonciation ; l'espace; les traits physiques, vestimentaires, langagiers ou onomastiques du personnage; la rhétorique et autres stratégies discursives (par exemple, l'éclatement des structures narratives et discursives dans $L a$ Québécoite, producteur d'un récit en miettes qui serait la mise en texte de l'altérité de la protagoniste). Pour la plupart, ces catégories où se rangent les différents procédés discursifs qui construisent l'altérité peuvent se passer de commentaire. L'énonciation, toutefois, jouit d'un statut particulier, car la question "Qui dit l'altérité ? " est fondamentale et mène à une distinction capitale, que Paterson emprunte à Simon Harel, entre deux types de récits :

1) celui où il y a mise à distance de l'Autre (une voix narrative le perçoit, le définit et le raconte comme dans le cas de la majorité des romans publiés au Québec avant les années 1960); 2) celui où l'Autre est le sujet énonçant (c'est le cas de beaucoup de récits migrants contemporains comme La Québécoite) (p. 29).

C'est cette distinction qui permettra à Paterson de poser par la suite que, depuis les années 1980 , la représentation de l'Autre se déroule selon deux axes différents. Il y a, d'une part, la littérature migrante qui donne la parole à l'Autre et, d'autre part, ce qu'elle appelle "le roman de l'altérité métissée, où l'Autre est une figure hétérogène, ambivalente, complexe " (p. 108) : "[s]e soustrayant à une structure binaire, l'Autre se dévoile dans sa complexité et ses contradictions. Ce faisant, c'est moins le rapport au Nous qui est dramatisé que la nature complexe de l'altérité $"$ (p. 136).

Cette bifurcation aurait d'ailleurs lieu dans le cadre d'une conception historique déjà binaire qui poserait

qu'il y a deux grandes étapes dans la représentation littéraire de l'Autre dans la littérature québécoise. La première, se manifestant depuis le début du roman jusqu'à la fin des années 1970, présente une figure de 
l'Autre comme objet du discours, par le biais d'une voix narrative à la troisième personne au sein d'un système binaire opposant le Nous et l'Autre. [...] La deuxième étape, qui se manifeste à partir des années 1980, est celle de la problématisation de la figure de l'Autre (p. 107-108).

Dans cette typologie, le fait énonciatif joue donc à deux reprises : la première grande étape de la représentation romanesque de l'Autre se caractériserait presque uniformément par une narration à la troisième personne qui construit l'Autre comme objet du discours, alors que la deuxième étape se manifesterait selon deux axes qui se distingueraient, en partie, par le système énonciatif utilisé - première ou troisième personne, l'Autre comme sujet ou comme objet du discours. Or, une telle typologie peut effectivement être utile, à condition de reconnaître qu'il s'agit là de dominantes et non pas de catégories étanches: La Québécoite relève bien de la littérature migrante qui donne la parole à l'Autre, mais c'est aussi, comme le sait très bien Janet Paterson, un " roman de l'altérité métissée, où l'Autre est une figure hétérogène, ambivalente, complexe ".

Les analyses de Paterson sont complétées, à la fin du livre, par un répertoire analytique, établi par Sandra Hobbs avec la collaboration de Kirsty Bell et Jeri English, des romans canadiens-français et québécois publiés entre 1846 et 1999 proposant un personnage principal qui est Autre par rapport à un groupe de référence. On peut consulter une version informatisée et plus complète de ce répertoire à l'adresse suivante : www.chass.utoronto.ca/french/alterite/. En plus des éléments de bibliographie et du bref résumé de l'intrigue qui figurent dans le répertoire, cette banque de données fournit des renseignements supplémentaires organisés sous les rubriques suivantes: "énonciation " (première ou troisième personne), "nom" (du personnage), "genre " (sexuel), "type d'altérité ", " groupe de référence ", " lieu du groupe de référence ", " marques d'altérité ", "valeur " (positive ou négative, avec explication). En combinant deux rubriques, par exemple "genre sexuel "et a type d'altérité " ou "énonciation " et "valeur ", le chercheur peut avoir accès à des données particulières, ce qui permet des recherches plus ciblées.

Cela dit, tout en reconnaissant la valeur indiscutable de ce répertoire en tant qu'instrument de travail, signalons quand même quelques-unes de ses limitations. Celles-ci sont de deux types: d'une part, il y a les 
limitations qui relèvent des critères d'inclusion et d'exclusion régissant le choix des textes (et des personnages) retenus; d'autre part, il y a celles qui relèvent plutôt de l'application de ces critères. Tout d'abord, seúls ont été retenus des romans littéraires qui ont déjà suscité une certaine attention critique; sont donc exclus tous les genres que l'on pourrait qualifier de paralittéraires - science-fiction, roman pour la jeunesse, roman populaire, fantastique, policier, etc. - ainsi que tout roman littéraire n'ayant suscité que peu d'attention critique (p. 175-176). Cela ne fait que souligner la portée résolument littéraire et du répertoire et de l'étude de Paterson. Les littéraires ne s'en plaindront sans doute pas, mais tout chercheur espérant profiter du répertoire ou de la banque de données pour mener à bien des recherches sur le phénomène plus généralement socioculturel de la représentation de l'Autre dans le roman québécois sera nécessairement déçu, d'autant plus que c'est souvent dans les genres paralittéraires ou "mineurs " que les attitudes sociales s'inscrivent en clair. Espérons donc que quelqu'un s'inspirera du travail déjà effectué par l'équipe de Paterson pour nous fournir ce répertoire complémentaire.

Les exclusions découlant de l'application des critères de sélection, surtout de ceux régissant la sélection des personnages, sont plus difficiles à repérer. Ne sont retenus que les personnages qui jouent un rôle important dans l'intrigue ; ceux quii sont considérés comme secondaires sont écartés. Là encore, c'est le littéraire qui semble l'emporter sur le socioculturel dans l'établissement des critères, mais, cette fois-ci, l'application de la règle implique nécessairement une interprétation ou un jugement de valeur, l'importance d'un personnage par rapport à l'intrigue étant toujours relative. D'ailleurs, dans la gamme des types d'altérité, on privilégie ceux qui relèvent de la "race " ou de la "nationalité " (263 personnages), les autres catégories figurant nettement moins souvent : "divers" (14), "étranger qui arrive dans un village" (17), "identité sexuelle " (4), " religion " (9), " santé mentale " (13). Cela nous laisse croire que les types d'altérité moins évidents, ou auxquels nous sommes aujourd'hui moins sensibilisés, comme ceux qui relèvent de la classe sociale, ne sont pas suffisamment représentés dans ce répertoire.

Chaque lecteur trouvera sans peine un certain nombre de personnages Autres qui ne figurent pas dans le répertoire ; je me limiterai à un seul, choisi pour la matrice discursive complexe dans laquelle s'inscrit son altérité. Le cas d'Alain Dubois, le personnage de Poussière sur la ville 


\section{RECENSIONS}

d'André Langevin, est d'autant plus intéressant qu'il s'insère dans un récit à la première personne où, en 1953 déjà, le narrateur-protagoniste se déclare Autre par rapport à un groupe de référence (les Macklinois), et cela dès le début du roman : " Je suis un intrus. [...] je n'ai rien à faire ici. [...] J'ai rêvé et, somnambule, je m'éveille dans la maison d'un autre ${ }^{2}$ ". Médecin d'origine québécoise, Dubois semble répondre de façon éloquente à l'attente suscitée par la mise en garde de Paterson quant au statut ontologique de l'Autre :

Tout lecteur, par exemple, qui tient pour acquis que l'Autre fictif appartient nécessairement à une catégorie regroupant des figures telles que celles de l'étranger, de l'Amérindien, du fou, du nomade, du criminel, reste bien en deçà de la subtilité du concept, de la variété et du potentiel de ses actualisations. Qui plus est, la précarité du statut ontologique de l'Autre est liée à la question des formes discursives. Car se méprendre sur l'essence de ce que représente l'Autre fictif, c'est ignorer la gamme de stratégies discursives opératoires dans la représentation littéraire (p. 17).

En effet, non seulement l'altérité de Dubois joue un rôle important tout au long du roman - sans elle, la décision de la fin perdrait tout son sens: "Je resterai. Je resterai, contre toute la ville. Je les forcerai à m'aimer ${ }^{3}$ " - mais encore, elle se trouve au centre d'un jeu discursif particulièrement intéressant. Car tout le conflit des codes si brillamment analysé dans ce roman par André Belleau ${ }^{4}$ tourne autour de la réflexion du docteur Dubois sur sa propre altérité, réflexion qui constitue le véhicule le plus important des échos intertextuels de Sartre et de Camus, et donc le signal du sérieux littéraire qui entre en conflit, dans le roman, avec la topographie simplifiée de Macklin, emblématique selon Belleau des codes socioculturels du Québec des années 1950. Ainsi, c'est donc à travers l'altérité du personnage de Dubois que s'inscrit l'altérité des codes littéraires importés. Or, si, comme le prétend Paterson, l'altérité est "la question la plus complexe de notre époquen (p. 15), le roman de

2. André Langevin, Poussière sur la ville, Montréal, Cercle du livre de France, 1953, p. 16.

3. Ibid., p. 213.

4. André Belleau, *Code social et code littéraiire dans le roman québécois ", L'Esprit créateur, vol. 23, n' 3, 1983, p. 19-31. 
Langevin, par la complexité discursive exemplaire de son déploiement de la figure, aura sans aucun doute un rôle clé à jouer dans une future histoire de la représentation de l'Autre au Québec.

Anthony Purdy

Université Western Ontario

\section{Dominique Marquis}

Un quotidien pour l'Église. L'Action catholique, 1910-1940.

Ottawa, Leméac, 2004.

Après l'incontournable essai sur l'Idéologie de L'Action catholique, réalisé par Richard Jones en 1974, il restait à décrire les conditions socioéconomiques de création, d'exploitation et de fonctionnement d'un journal qui, peu avant la Deuxième Guerre mondiale, connaissait un tirage de plus de 52000 exemplaires. C'est désormais chose faite grâce à l'ouvrage que Dominique Marquis a tiré de sa thèse de doctorat en histoire présentée à l'Université du Québec à Montréal. Pour ce faire, elle s'est appuyée sur de précieuses archives jusqu'à présent peu exploitées, à savoir les fonds de l'Action sociale catholique de l'archevêché de Québec, de la chancellerie de l'archevêché de Montréal et des Archives nationales du Québec, qui contiennent des dossiers sur L'Action catholique, et sur les papiers d'Eugène L'Heureux détenus par les Archives de l'Université Laval. Elle a également utilisé la base de données sur la presse québécoise Hyperbec, ce qui lui a permis de dresser des constats et comparaisons statistiques très utiles.

À partir d'une analyse minutieuse du contenu, de la mise en pages et de la stratégie publicitaire du quotidien, l'historienne décrit le processus suivi par l'Église pour participer à la culture de masse émergente. Habituée au journalisme de combat du xIX siècle, l'institution doit désormais compter sur un mode de communication différent, celui de la presse d'information du $\mathrm{xx}^{\mathrm{e}}$ siècle. S'adapter implique d'adopter un système et une pratique que les quotidiens concurrents - pour ne pas dire "adversaires" - manient avec efficacité. Bien que très réticents au début, 